\title{
Role of Plasma Amino Acids Profile in Pathogenesis and Prediction of Severity in Patients with Drug Resistant Epilepsy \\ TahiaH. Saleem ${ }^{1}$, Ahmed Y. Nassar ${ }^{1}$, Hamdy N. El-Tallawy ${ }^{2}$, Sara A. Atta ${ }^{1}$,Marwa A. Dahpy ${ }^{1 *}$ \\ ${ }^{1}$ Medical Biochemistry and Molecular biology Department, Faculty of Medicine, Assiut University, \\ ${ }^{2}$ Neurology and Psychiatry Department, Faculty of Medicine, Assiut University, Assiut, Egypt. \\ *Corresponding author: MarwaA. Dahpy. Email: marwadahpy@yahoo.com, marwadahpy@aun.edu.eg
}

\begin{abstract}
Background: Abnormal plasma levels of free amino acids may predict the severity in patients with drug resistant epilepsy (PRE), having probability to affect their therapeutic approach.

Objectives:We aimed to illuminate the effect of plasma free amino acids (PFAAs) profiles on the etiology of patients with PRE and their contribution on the frequencies of epileptic fits.

Patients and Methods:We collected clinical and metabolomic data of 90 subjects; 45 of them were PRE patients, and other 45 age and sex matched healthy controls. Quantitative measurements of PFAAs profiles using SykamAutomatic Amino Acid Analyzer S433, in addition to fasting blood sugar, liver function tests, kidney function tests and lipid profile also, were determined.

Results: The plasma levels of glutamate, glycine and Gamma amino butyric acid(GABA) plasma levels were significantly increased in PRE group compared to controls $(\mathrm{p}<0.0001)$, their plasma levels also showed significant increase with increased frequency of epileptic fits. Plasma leucine, phenylalanine, aspartate ,ornithine, citrulline, serine and alanine levels $(p<0.0001)$ were significantly increased in PRE group in relation to healthy controls. Interestingly, on the other hand, histidine, isoleucine, lysine, threonine and the amino acid derivative taurine levels were significantly decreased in PRE patients compared to healthy controls.

Conclusion:Few biomarkers of PRE are available to find the severity and rate of progressionof PRE. The present study showed that altered plasma amino acids and their derivatives may be candidate markers for PRE, help explaining its pathogenesis, and for further researches concerning normalization of the disturbed amino acid/s or its derivative/s (GABA and taurine) in managing PRE patients.
\end{abstract}

Keywords: Aminogram; Plasma free amino acid profile; Pharmacoresistant epilepsy, amino acids.

Abbreviations:Anti-epileptic drugs (AEDs), Branched-chain amino acids (BCAAs), Plasma free amino acids (PFAAs),Pharmaco-resistant epilepsy (PRE).

\section{INTRODUCTION}

Epilepsy is the most common chronic neurological disease. It affects over 70 million people worldwide ${ }^{(1)}$ with increased risk of mortality, and socioeconomic consequences that impairing quality of life ${ }^{(2)}$. It is diagnosed with either recurrent two or more unprovoked seizures occurring at least 24 hours apartor a single seizure, accompanied by evidence from clinical, or neuroimaging tests that an increased risk exists for future seizures over the next 10 years ${ }^{(\mathbf{3})}$. Approximately $40 \%$ of patients with epilepsy exhibit resistance to pharmaco therapy over the past two decades ${ }^{(4)}$.Drug or pharmaco resistant epilepsy (PRE) is defined as the failure of tolerable trials of two tolerated and suitably chosen mono therapies singly or in combination to achieve sustained seizure freedom for more than one year ${ }^{(5)}$. PRE patients have increased risk of mortality due to sudden unexpected death in epilepsy ${ }^{(6)}$.

Amino acids not only are dynamic structural building blocks of proteins but are also active signaling molecules regulating metabolism.

Several neurotransmitters in the brain synthesized from amino acids. The concentration of amino acids in plasma regulate the invivo synthesis of individual amino acids in the brain so it can affect the brain functions ${ }^{(7)}$.
Glutamate is the most abundant excitatory neurotransmitter in the brain, which means increases the probability that the neuron will have an action potential ${ }^{(\mathbf{8})}$.Glutamate action mediated through activation of $\mathrm{G}$ protein coupled metabotropic receptors and ionotropic receptors so, altered glutamate amino acid transport or metabolism may be a contributory factor in some genetic and acquired forms of epilepsy ${ }^{(9)}$.

Inhibitory neurotransmission is mediated by several neurotransmitters, as GABA and glycine ${ }^{(\mathbf{1 0 )}}$. Gamma amino butyric acid (GABA)increases the permeability of depolarized membranes to ions which leads to acceleration of return to resting potential of all membrane segments. It is also, decreasing membrane sensitivity to stimulation so, stabilizes undepolarized membrane segments. Functional and structural alterations of GABAergic pathway participate to the pathophysiology of many brain disorders including epilepsy ${ }^{(\mathbf{1 1})}$.

The essential branched-chain amino acids BCAAs (leucine, isoleucine, and valine) have lately used as a treatment for PRE. Circulating BCAAs can readily enter the brain, where they supply glutamate biosynthesis and may either suppress or prompt acute seizures ${ }^{(\mathbf{1 2})}$. 
Homocysteine and taurine are considered to be neurotransmitters and neuromodulators. Homocysteine has a hyperexcitability role, while taurine has inhibitory and neuroprotective properties (13). After acute epilepsy seizures, amino acid changes in the cerebrospinal fluid (CSF) were observed and may be limited to a reduction in the level of taurine ${ }^{(\mathbf{1 4})}$. The taurine neuroprotective effect is linked with its anticonvulsive properties, it is mediated by its interactions with glutamate, GABA pathway and oxidative stress ${ }^{(15)}$.

Aromatic amino acids mainly phenylalanine can be implicated in neuronal survival and differentiation through regulating expression of brain-derived neurotrophic factors ${ }^{(16)}$.

\section{MATERIALS AND METHODS}

The current study is a case-control study that was conducted on 45 patients with PRE (group A)who were selected from the outpatients and inpatients of Epilepsy clinic, Neurology and psychiatry Department of Assiut University Hospital, together with, 45 age and sex matched healthy persons(controls, group B) who were selected randomly from the same population as the patients, with no known personal history or family history of epilepsy or any neurological or psychiatric diseases, they were presented to the outpatients clinic with non-specific complaints. The study in collaboration with Metabolic and Genetic Disorders Unit (MGD) in the period from March 2017 to July 2018, Faculty of Medicine, Assiut University, Egypt.

Ethical approval: The study protocol was approved by the Ethics Committee of Faculty of Medicine, Assiut University (code number: IRB00008718).

All participating subjects gave written informed consent after the purpose and method of the study were briefly discussed to each participant.

Diagnosis of PRE was depending on the presence of uncontrolled seizures with an average frequency of one fit per month for two years with usage of at least three different tolerated and appropriately chosen anti-epileptic drugs (AEDs) (singly or in combination) ${ }^{(17)}$. Group (A) patients were receiving valproic acid, clonazepam and oxycarbazine in different combinations.

Epileptic patients with other types of neurological diseases, chronic medical diseases (cardiac, hepatic or renal or malignancies or those currently pregnant or breast-feeding) all were excluded from the study.

\section{Data collection, Clinical examination and Imaging studies:}

Personal data including age, residence and occupation, handedness, smoking or drug addiction, family history of epilepsy, consanguinity between parents, history of febrile convulsions, personal or family history of migraine, history of head trauma orany CNS infections, detailed history of epileptic fits, past and current medications, and careful therapeutic history together with detailed clinical examination were taken by a neurologist.

An Electroencephalography (EEG) was performed by specialized neurologist, additionally, magnetic resonance imaging (MRI) was done when indicated.

\section{Laboratory workup:}

After an overnight fasting, $4 \mathrm{ml}$ of antecubital venous blood samples were drawn from patients and controls. The blood samples were collected in heparinized tubes, Plasma products were separated by centrifugation of blood samples at $3000 \mathrm{rpm}$ for $10 \mathrm{~min}$, then stored at $-20^{\circ} \mathrm{C}$ for measurement of the PFAA profiles.

Ion exchange separation method using high performance liquid chromatography using a Sykam Automatic Amino Acid Analyzer S433 supplied by Sykam GmbH, Germany (catalog no. 1120001).Free amino acid samples were prepared from plasma by acidic protein precipitation, where $200 \mu \mathrm{l}$ of $10 \%$ sulfosalicylic acid solution was added to $800 \mu \mathrm{l}$ plasma, mixed by vortex, then solution was allowed to cool down at about $4{ }^{\circ} \mathrm{C}$ for $30 \mathrm{~min}$, and then was centrifuged for $10 \mathrm{~min}$ at $14000 \mathrm{rpm}$. Supernatant liquor was diluted with same amount of sample dilution buffer (catalog no. S000015). One hundred $\mu 1$ of each of prepared samples and ready to use amino acid physiological standard (Catalog no. 6006005) wereinjected directly.

Cation separation column LCAK06/Li was used (catalog no. 5112008 ) with size: $150 \mathrm{~mm} \times 4.6 \mathrm{~mm}$, specification range: met efficiency: $>48000$, asymmetry: $0.8-1.5$, resolution THR/SER: > 1700, and column pressure: 45-80bar. Buffer: Sykam LiA1,LiB-1,LiC-4.The ready to use ninhydrin reagent (catalog no. S000025) and citrate buffers in different $\mathrm{pH}(2.9,4.2$, and 8.0) were used. Analysis at wave length 440nm:570 $\mathrm{nm}$. The sample chromatogram is compared to the standard measurements curve (Fig:1A) to obtain various amino acid values, then results were multiplied by a dilution factor of $2.5^{(\mathbf{( 1 8 )}}$.

Routine blood investigation including: fasting blood glucose, liver function, kidney function tests and lipid profile were analyzed using enzyme colorimetric kits supplied by Biodiagnostics, Egypt. Additionally, blood level of anti-epileptic drugs were measured for the included patients at Assiut University Hospitals, Egypt.

\section{Statistical Analysis}

Data were collected and analyzed using SPSS (Statistical Package for the Social Science) version 20 . Continuous data was expressed as mean \pm SD or median (range), while nominal data was expressed as frequency (percentage).Student's $t$ test tested 
statistical significance for two groups and ANOVA test for threegroups.

\section{RESULTS}

The mean age of the patients (group A) was 22.56 \pm 9.45 years while that of healthy control group (group B) were $22.09 \pm 6.78$ years $(\mathrm{P}=0.98)$. Duration of Epilepsy for patients (group: A) ranged from 2-26 years. Both of patients and control groups included 29 males and 16 females. Regarding risk factors in patients; consanguinity was positive in $26(57.8 \%)$ of patients. Positive family history of epilepsy found in $18(40 \%)$ of patients, while history of febrile convulsion presented in two patients only $(4.4 \%)$.

General biochemical studies showed non-significant difference regarding serum glucose, lipid profile, liver or renal functions.

Concerning the age ofonset, it was noticed that age of onset of the majority about $35.6 \%$ of patients were at adolescence (12-18 year) followed by $20 \%$ at early adulthood (>18 year), 20\% at late childhood (612 year), $13.3 \%$ Early childhood (1- 6 year), and $11.1 \%$ at infancy $(<1$ year). Of patients $55.6 \%$ and $42.2 \%$ had daily (severe), and weekly (moderate) seizures respectively. Only one case $(2.2 \%)$ was mildly affected and had a monthly fits. Serial fits presented in 35 (77.8\%) patients while 9 (20\%) patients had history of status epilepticus.

The plasma free amino acids levels in the study groups have been shown in (Table 1\& Fig.1B \&C). Plasma amino acids profile in patients and controls showed interesting significant differences, where plasma levels of glutamate, glycine, leucine, phenylalanine, aspartate, ornithine, citrulline,serine and alanine levels and amino acid derivative GABA,were all increased in PRE group compared to controls $(p<0.0001)$. On the other hand, plasma levels

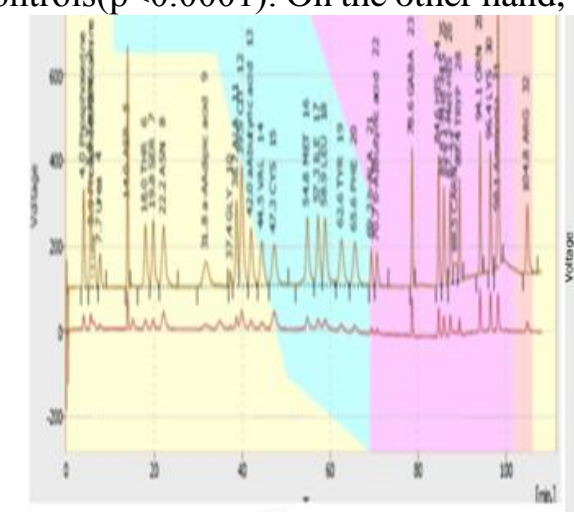

A

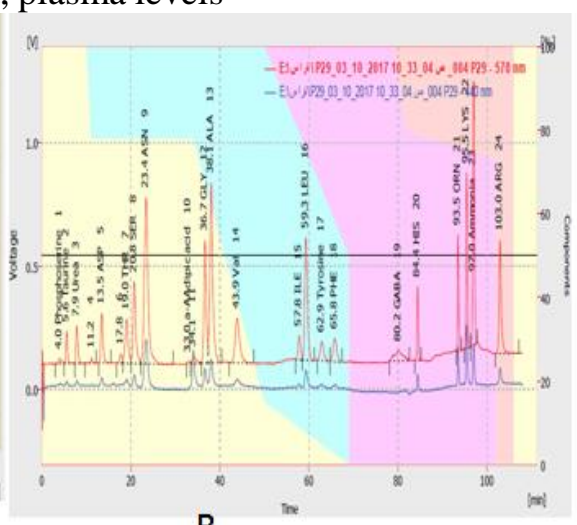

B of histidine, isoleucine, lysine, threonine and the amino acid derivative taurine levels were significantly decreased in PRE patients compared to controls ( $\mathrm{p}<0.0001$ each).

Regarding the frequency of epileptic fits, the severely affected patients (those complained of fit daily) had significantly higher glutamate, glycine and GABA plasma levels (P-value $<0.000$ each) compared to moderately affected patients (table 2), their serum ornithine, aspartate and alanine also showed also, significant increase in severely affected PRE patients. On the contrary, serum phenylalanine showed significant decreased in severely affected PRE patients compared to those moderately affected.

Subgrouping PRE patients according to presence or absence of status epileptics, (table: 3) showed also significant higher levels of glutamate amino acid in patients with recurrent attacks of status epileptics ( $\mathrm{p}$-values $=0.023$ ).

We further subdivide the patient group $(n=45)$ into five subgroups according to age of onset of epilepsy.We found no significant difference either in the plasma amino acid profiles between the subgroups (except for arginine amino acid)(Table:4) nor, in the correlation studies between plasma amino acid profiles and age of onset)(results not showed).

Correlation studies between frequencies of fits and levels of PFAA, confirmed previous results and showed significant positive correlation among GABA, glycine, glutamate, aspartate and alanine amino acids and the frequency of fits, although, significant negative correlation found between the frequency of fits and only phenylalanine amino acid(table:5) .

Fig 1: Chromatogram of amino acids Aminogram.(A): standard curve: Analysis at wavelength $440 \mathrm{~nm}: 570 \mathrm{~nm}(\mathrm{~B})$ for the studied patients.(C) for the included controls (group B).Abbreviations:-ASP:Aspartate; Ther:Threonine; Ser:Serine; GlY:Glycine; Ala:Alanine; Val:Valine; Met:Methionine; Ile:Isoleucine; Leu:Leucine; PHE:Phenylalanine; GABA:Gamma amino butyric acid; Orn:Ornithine; LYS:Lysine; 8: Glutamate.

Table 1: Plasma free amino acid levels $(\mu \mathrm{mol} / \mathrm{L})$ in the studied groups (patients and controls).

\begin{tabular}{|c|c|c|c|}
\hline Variables & Patients $(A) \mathbf{N}=45$ & Healthy control $(B) \mathbf{N}=45$ & P value \\
\hline
\end{tabular}




\begin{tabular}{|c|c|c|c|}
\hline \multicolumn{4}{|c|}{ Branched chain Amino Acids (BCAAs) } \\
\hline Valine & $394.86 \pm 98$ & $401 \pm 51.5$ & 0.6718 \\
\hline Isoleucine & $145.26 \pm 8$ & $220.2 \pm 7.3$ & 0.0001 \\
\hline Leucine & $492.79 \pm 123$ & $180.8 \pm 19.66$ & 0.0001 \\
\hline \multicolumn{4}{|c|}{ Aromatic Amino Acids (ARAAs) } \\
\hline Tyrosine & $137.7 \pm 7.9$ & $141.66 \pm 6.7$ & 0.8 \\
\hline Phenylalanine & $198.5 \pm 8$ & $82.96 \pm 6$ & 0.0001 \\
\hline Histidine & $134.5 \pm 5.16$ & $262.3 \pm 35.3$ & 0.0001 \\
\hline \multicolumn{4}{|c|}{ Excitatory Amino Acid } \\
\hline Glutamate & $280.8 \pm 70$ & $155 \pm 20$ & 0.0001 \\
\hline Aspartate & $192 \pm 18$ & $26.9 \pm 15.3$ & 0.0001 \\
\hline \multicolumn{4}{|c|}{ Amino Acid derivatives } \\
\hline Urea & $438.76 \pm 7$ & $402.4 \pm 6.8$ & 0.0515 \\
\hline GABA & $193 \pm 3.7$ & $67.86 \pm 1.8$ & 0.0001 \\
\hline Taurine & $47.71 \pm 1.9$ & $83 \pm 1.7$ & 0.0001 \\
\hline \multicolumn{4}{|c|}{ Other Amino Acid } \\
\hline Ornithine & $238.8 \pm 59$ & $150.2 \pm 35$ & 0.0001 \\
\hline Lysine & $424.95 \pm 106$ & $521.8 \pm 130$ & 0.0002 \\
\hline Arginine & $348.7 \pm 87$ & $406.2 \pm 101.9$ & 0.0045 \\
\hline Citrulline & $116 \pm 27.7$ & $28.6 \pm 1.59$ & 0.0110 \\
\hline Methionine & $33.8 \pm 4.49$ & $37.9 \pm 3.7$ & 0.1637 \\
\hline Threonine & $214.5 \pm 7.5$ & $290.8 \pm 55.6$ & 0.0001 \\
\hline Serine & $368.87 \pm 85.6$ & $201.3 \pm 12$ & 0.0001 \\
\hline Glycine & $575.4 \pm 46.9$ & $277.6 \pm 20.7$ & 0.0001 \\
\hline Alanine & $470.8 \pm 33.5$ & $282.6 \pm 22$ & 0.0001 \\
\hline
\end{tabular}

Data represented as mean $(\mu \mathrm{mol} / \mathrm{l}) \pm S D$, comparison between the two subgroups by using T. Test. $P<0.05$ : significant $P<0.01$. Significant results are bold and gray shading.

Table 2, The levels of plasma free amino $\operatorname{acid}(\mu \mathrm{mol} / \mathrm{l})$ in patients $\operatorname{group}(\mathrm{A}) \operatorname{according}$ to frequency of epileptic fits

\begin{tabular}{|l|c|c|c|}
\hline \multirow{2}{*}{ Variables } & \multicolumn{2}{|c|}{ Frequency of Fits } & \multirow{2}{*}{ P-value } \\
\cline { 2 - 4 } & Moderate(weekly) $\mathbf{n = 1 9}$ & Severe (daily) $\mathbf{n = 2 5}$ & \\
\hline GABA & $117.23 \pm 2.74$ & $163.76 \pm 5.91$ & $\mathbf{0 . 0 1 0 6}$ \\
\hline Valine & $402.32 \pm 7.76$ & $421.26 \pm 12.99$ & 0.563 \\
\hline Isoleucine & $150.35 \pm 7.53$ & $156.11 \pm 6.52$ & 0.814 \\
\hline Leucine & $452.8 \pm 11.9$ & $504.73 \pm 48.89$ & 0.542 \\
\hline Tyrosine & $151.9 \pm 7.45$ & $163.57 \pm 8.93$ & 0.540 \\
\hline Phenylalanine & $216.9 \pm 78.93$ & $159.48 \pm 8.3$ & $\mathbf{0 . 0 2 4}$ \\
\hline Histidine & $152.71 \pm 7.71$ & $185.39 \pm 9.27$ & 0.216 \\
\hline Glutamate & $166.21 \pm 24.62$ & $253.52 \pm 55.05$ & $\mathbf{0 . 0 0 0}$ \\
\hline Aspartate & $135.63 \pm 9.59$ & $189.3 \pm 3.13$ & $\mathbf{0 . 0 5 0}$ \\
\hline Glycine & $325.75 \pm 38.6$ & $526.49 \pm 39.31$ & $\mathbf{0 . 0 0 0}$ \\
\hline Ornithine & $263.28 \pm 20.95$ & $265.83 \pm 19.58$ & $\mathbf{0 . 0 0 2}$ \\
\hline Lysine & $412.96 \pm 41.11$ & $416.72 \pm 79.81$ & 0.940 \\
\hline Arginine & $407.14 \pm 58.83$ & $377.64 \pm 79.91$ & 0.574 \\
\hline Taurine & $122.4 \pm 5.05$ & $116.07 \pm 4.6$ & 0.645 \\
\hline Citrulline & $155.52 \pm 34.08$ & $69.47 \pm 9.44$ & 0.080 \\
\hline Methionine & $32.26 \pm 8.4$ & $32.89 \pm 8.31$ & 0.805 \\
\hline Threonine & $323.08 \pm 15.29$ & $313.53 \pm 51.25$ & 0.815 \\
\hline Serine & $437.35 \pm 85.67$ & $457.43 \pm 69.68$ & 0.618 \\
\hline Alanine & $353.9 \pm 177.27$ & $445.81 \pm 18.74$ & $\mathbf{0 . 0 4}$ \\
\hline
\end{tabular}

Data represented as mean $(\mu \mathrm{mol} / \mathrm{l}) \pm S D$, comparison between the two subgroups by using T. Test: $P<0.05$ : significant Nb: Number of epileptic patients $=45$, only one patient was mildly affected (suffered of epileptic fits once monthly). This patient was not included in this statistic, so number of patients in table (2) were 44 patients. Significant results are bold and gray shading.

Table 3: Comparison of the levels of plasma free amino acid $(\mu \mathrm{mol} / \mathrm{L})$ in Epileptic patients $\operatorname{group}(\mathrm{A})(\mathrm{N}=45)$ according to presence or absence of status epileptics 
ejhm.journals.ekb.eg

\begin{tabular}{|l|c|c|l|}
\hline & Present (n=9) & Absent (n=36) & \multirow{2}{*}{} \\
\cline { 2 - 3 } & Mean \pm SD & Mean \pm SD & \\
\hline GABA & $143.16 \pm 4.81$ & $143.9 \pm 6.85$ & 0.974 \\
\hline Valine & $377.01 \pm 88.39$ & $417.2 \pm 11.95$ & 0.323 \\
\hline Isoleucine & $162.72 \pm 3.47$ & $151.23 \pm 5.35$ & 0.698 \\
\hline Leucine & $382.59 \pm 95.62$ & $450.08 \pm 132.23$ & 0.154 \\
\hline Tyrosine & $134.33 \pm 2.42$ & $161.89 \pm 6.61$ & 0.256 \\
\hline Phenylalanine & $143.44 \pm 6.48$ & $196.3 \pm 4.11$ & 0.094 \\
\hline Histidine & $189.26 \pm 14.97$ & $165.3 \pm 8.82$ & 0.458 \\
\hline Glutamate & $256 \pm 44.81$ & $202.56 \pm 50$ & $\mathbf{0 . 0 0 5}$ \\
\hline Aspartate & $143.87 \pm 4.36$ & $168.74 \pm 8.01$ & 0.470 \\
\hline Glycine & $457.07 \pm 43.71$ & $426.05 \pm 84.19$ & 0.641 \\
\hline Ornithine & $229.84 \pm 31.05$ & $269.85 \pm 16.39$ & 0.373 \\
\hline Lysine & $450.48 \pm 55.81$ & $406.17 \pm 62.67$ & 0.465 \\
\hline Arginine & $321.65 \pm 88.97$ & $403.88 \pm 62.41$ & 0.195 \\
\hline Taurine & $104.33 \pm 26.14$ & $123.36 \pm 27.24$ & 0.059 \\
\hline Citrulline & $108.55 \pm 9.62$ & $118.99 \pm 25.86$ & 0.243 \\
\hline Methionine & $31.02 \pm 4.13$ & $33.03 \pm 8.89$ & 0.515 \\
\hline Threonine & $272.71 \pm 61.67$ & $325.7 \pm 81.61$ & 0.07 \\
\hline Serine & $415.63 \pm 79.8$ & $452.88 \pm 74.78$ & 0.572 \\
\hline Alanine & $400.05 \pm 55.17$ & $403.69 \pm 63.55$ & 0.948 \\
\hline
\end{tabular}

By using Independent Samples. Significant results are bold and gray shading.

Table 4: Plasma free amino acid levels $(\mu \mathrm{mol} / \mathrm{l})$ in patient group $(\mathrm{A})(\mathrm{N}=45)$ according to age of onset of epilepsy.

\begin{tabular}{|c|c|c|c|c|c|c|}
\hline \multirow{3}{*}{ Variables } & \multicolumn{5}{|c|}{ Age at onset } & \multirow{3}{*}{ P. value } \\
\hline & $\begin{array}{l}\text { Infancy } \\
(n=5)\end{array}$ & $\begin{array}{l}\text { Early } \\
\text { childhood }(n=6)\end{array}$ & $\begin{array}{l}\text { Late } \\
\text { childhood (n=9) }\end{array}$ & $\begin{array}{l}\text { Adolescence } \\
(\mathrm{n}=16)\end{array}$ & $\begin{array}{l}\text { Early } \\
\text { adulthood (n=9) }\end{array}$ & \\
\hline & Mean \pm SD & Mean \pm SD & Mean \pm SD & Mean \pm SD & Mean \pm SD & \\
\hline GABA & $124.47 \pm 5.93$ & $108.81 \pm 8.04$ & $183.06 \pm 9.05$ & $152.24 \pm 6.67$ & $123.39 \pm 5.48$ & 0.097 \\
\hline Valine & $431.4 \pm 7.15$ & $395.76 \pm 9.54$ & $456.61 \pm 7.22$ & $406.2 \pm 35.77$ & $363.55 \pm 9.64$ & 0.469 \\
\hline Isoleucine & $159.08 \pm 5.29$ & $216.13 \pm 28.26$ & $145.71 \pm 7.71$ & $136.41 \pm 7.68$ & $146.97 \pm 9.07$ & 0.318 \\
\hline Leucine & $492.43 \pm 62.64$ & $551.22 \pm 44.86$ & $379.5 \pm 66.01$ & $570.01 \pm 38.19$ & $356.99 \pm 23.91$ & 0.275 \\
\hline Tyrosine & $154.53 \pm 8.16$ & $159.16 \pm 6.87$ & $169.66 \pm 6.1$ & $161.98 \pm 8.76$ & $132.31 \pm 4.17$ & 0.793 \\
\hline Phenylalanine & $162.83 \pm 6$ & $185.91 \pm 9.57$ & $201.49 \pm 8.32$ & $204.34 \pm 9.67$ & $149.5 \pm 5.88$ & 0.557 \\
\hline Histidine & $245.84 \pm 7.65$ & $140.32 \pm 9.42$ & $137.53 \pm 8.23$ & $167.52 \pm 8.09$ & $184.97 \pm 11.59$ & 0.179 \\
\hline Glutamate & $222.2 \pm 58.6$ & $224.5 \pm 57.18$ & $248 \pm 62.58$ & $200.69 \pm 7.68$ & $198.33 \pm 8.29$ & 0.618 \\
\hline Aspartate & $147.64 \pm 6.4$ & $107.91 \pm 28.67$ & $154.4 \pm 3.79$ & $173.35 \pm 10.01$ & $202.29 \pm 18.27$ & 0.377 \\
\hline Glycine & $502.55 \pm 17.3$ & $436 \pm 17.29$ & $507.93 \pm 15.4$ & $389.35 \pm 20.3$ & $391.31 \pm 57.04$ & 0.432 \\
\hline Ornithine & $258.15 \pm 34.06$ & $190.44 \pm 9.37$ & $351.16 \pm 12.4$ & $249.48 \pm 17.19$ & $244.18 \pm 27.23$ & 0.100 \\
\hline Lysine & $456.81 \pm 16.99$ & $412.26 \pm 71.59$ & $365.31 \pm 55.07$ & $431.79 \pm 62.61$ & $413.59 \pm 99.04$ & 0.861 \\
\hline Arginine & $308.28 \pm 14.68$ & $246.62 \pm 15.83$ & $504.44 \pm 49.12$ & $392.61 \pm 48.39$ & $399.09 \pm 70.96$ & 0.039 \\
\hline Taurine & $103.41 \pm 25.29$ & $126.48 \pm 31.68$ & $96.17 \pm 8.89$ & $134.75 \pm 5.31$ & $120.27 \pm 5.78$ & 0.270 \\
\hline Citrulline & $35.86 \pm 2.46$ & $144.98 \pm 36.89$ & $89.34 \pm 4.36$ & $112.5 \pm 27.72$ & $118.6 \pm 11.1$ & 0.842 \\
\hline Methionine & $35.66 \pm 4.91$ & $30.4 \pm 8$ & $35.69 \pm 8.2$ & $30.88 \pm 5.12$ & $32.49 \pm 8.53$ & 0.553 \\
\hline Threonine & $319.52 \pm 6.25$ & $308.28 \pm 9.74$ & $333.19 \pm 20.37$ & $329.55 \pm 74.82$ & $273.43 \pm 12.07$ & 0.875 \\
\hline Serine & $443.33 \pm 82.55$ & $401.31 \pm 40.2$ & $531.47 \pm 73.97$ & $451.35 \pm 88.26$ & $379.45 \pm 63.7$ & 0.436 \\
\hline Alanine & $455.94 \pm 69.68$ & $370.92 \pm 89.87$ & $462.11 \pm 10.5$ & $404.14 \pm 69.42$ & $333.65 \pm 39.07$ & 0.371 \\
\hline
\end{tabular}

By using one-way ANOVA test; $P>0.05$ : non-significant, Boldface: $P<0.05$ : significant.Significant results are bold and gray shading.

Table 5: Correlation between frequencies of fits and all quantitative parameters in Patients (group A) $(\mathrm{N}=45)$

\begin{tabular}{|l|c|c|}
\hline \multirow{2}{*}{ Variables } & \multicolumn{2}{|c|}{ Frequency of fits } \\
\cline { 2 - 3 } & $\mathbf{r}$ & $\mathbf{P}$ \\
\hline Age at onset & -0.117 & 0.443 \\
\hline
\end{tabular}




\begin{tabular}{|l|l|l|}
\hline GABA & $\mathbf{0 . 3 5 5}^{*}$ & $\mathbf{0 . 0 1 7}$ \\
\hline Valine & 0.173 & 0.255 \\
\hline Isoleucine & -0.018 & 0.906 \\
\hline Leucine & 0.191 & 0.209 \\
\hline Tyrosine & 0.146 & 0.339 \\
\hline Phenylalanine & $\mathbf{- 0 . 4 2 4}^{* *}$ & $\mathbf{0 . 0 0 4}$ \\
\hline Histidine & 0.214 & 0.158 \\
\hline Glutamate & $\mathbf{0 . 6 8 5}^{* *}$ & $\mathbf{0 . 0 0 0}$ \\
\hline Aspartate & $\mathbf{0 . 3 5 9}^{*}$ & $\mathbf{0 . 0 1 5}$ \\
\hline Glycine & $\mathbf{0 . 5 8 9}^{* *}$ & $\mathbf{0 . 0 0 0}$ \\
\hline Ornithine & 0.089 & 0.562 \\
\hline Lysine & 0.092 & 0.549 \\
\hline Arginine & -0.041 & 0.788 \\
\hline Taurine & -0.070 & 0.647 \\
\hline Citrulline & -0.041 & 0.790 \\
\hline Methionine & 0.034 & 0.826 \\
\hline Threonine & 0.062 & 0.687 \\
\hline Serine & 0.139 & 0.363 \\
\hline Alanine & $\mathbf{0 . 3 4 3}$ & $\mathbf{0 . 0 2 1}$ \\
\hline
\end{tabular}

By using Spearman's correlation. Significant results are gray shading and boldface: * Statistically significant correlation $(p<0.05), * *$ Statistically significant correlation $(p<0.01)$.

\section{DISCUSSION}

Amino Acid profile in plasma and CSF give strong indication for excitability state and health of the brain. In the present study levels of the excitatory amino acids glutamate in addition to aspartate amino acid were significantly increased in PRE patients compared to healthy controls, also their levels increase significantly with increased frequency of fits.Aspartate may be a key excitatory neurotransmitter besides glutamate in pathogenesis of epilepsy, this in accordance with previous study of Huxtable et al.$^{(\mathbf{1 9 )})}$ that concluded that fasting plasma levels of aspartate and glutamate significantly increase in epilepsy.

This also, observed by Janjua et al. ${ }^{(\mathbf{2 0})}$ who found these amino acids act as excitatory neurotransmitter playing a key role in the pathogenesis of PRE epilepsy and are also compatible with neurochemical and neurophysiological evidence implicating glutamic acid in the mechanism of seizure. Also, this increases in aspartate and glutamate concentrations may be explained by the prescription of phenytoin as mentioned by Huxtable et al. $^{\left({ }^{19}\right)}$.

Regarding inhibitory amino acid glycine, it shows a significant increase in patients as compared to controls. This can be explained by either the administration of valproate which increases the plasma levels of glycine (by inhibition of the glycine cleavage system), or defect in receptor and signaling pathway in the brain as documented by Rainesaloet al. ${ }^{(14)}$.

The current study revealed that plasma levels of GABA showed a significant increase in PRE patients compared to healthy controls (P-value $<0.0001)$ which can be explained by the administration of anti-epileptic drugs that increase local GABA concentration and another explanation is resistance of GABA receptors to central GABA that can be reflected in part in by elevated plasma GABA. That was in accordance with a previous study by Löscher et al. ${ }^{(21)}$ who demonstrated in animal study that increased central GABA concentration by anticonvulsant inhibitors of GABA catabolism are reflected in part in plasma GABA.These results suggest that the GABA in plasma may be useful to indirectly monitor the effect of such drugs on brain GABA concentration in humans.

The role of BCAA on human brain is somewhat controversy. Current study showed significant increase of levels of leucine in patients with PRE compared to healthy controls, the finding that may partially explained by that of Evangeliou et al. (22) who concluded that constant oral supplementation of BCAAs worsens seizure propagation and causes neuron loss in animals with mesial temporal lobe epilepsy.

Although, phenylalanine concentration is mildly significantly elevated in patients compared to controls. Yet, its levels were negatively correlated with age of onset, this in accordance with Huxtable et $\boldsymbol{a l} .{ }^{(\mathbf{1 9 )}}$, who found decreased concentrations of aromatic amino acids as tyrosine and phenylalanine in plasma after acute seizures.

Suárez et al. ${ }^{(23)}$ stated that the biosynthesis of taurine is depending on nutritional state, protein intake, and cysteine accessibility, so it recorded highly variable among individuals. In current study, taurine level was decreased in patients compared to controls, this finding may be due to the lack of neuroprotective role of taurine in epileptic patients as explained by Hrncic et al ${ }^{(\mathbf{1 3})}$.

Our results of significantly decrease histidine amino acid in PRE patients than controls is in accordance with previous work by Gietzen $\boldsymbol{e t} \boldsymbol{a l} .{ }^{(24)}$, who demonstrated that deficiencies of histidine exacerbate seizures, furthermore, limitation of histidine amino acid may excite the anterior pyriform cortex of the cerebrum, which contains a zone of high epileptogenicity.

Arginine amino acid showed tend to significant increase in PRE patients compared to controls this finding in accordance with Carlson et $\boldsymbol{a l} .{ }^{(25)}$ who showed five times increase in arginine levels in patients with focal epilepsy. De Sarroet al. ${ }^{(26)}$ results also support contribution of arginine to the origins of seizure activity through the action of nitric oxide which is formed from L-Arginine upon excitatory amino acid receptor activation within the prepiriform cortex.

The study has public health implications, as according to Kobow and Blümcke ${ }^{(27)}$, about 50 million people worldwide have epilepsy, thus its considered the most common worldwide chronic and severe neurological disease. Above $30 \%$ of epileptic patients have insufficient control of their seizures with pharmacological therapy. In addition, few biomarkers of PRE are available to assess the severity and rate of progression. The present study showed that plasma amino acids and their derivatives may be a candidate 
marker for selection in future risk scores for PRE if larger sample size studies can also replicate these findings.

\section{CONCLUSION}

Significant differences among plasma amino acids profile between patients and controls establish an interesting connection between plasma amino acid changes and pathogenesis of PRE.

Identification of PFAA changes may contribute to drug failure in PRE, allowing better understanding of its cellular and metabolic mechanisms and is critical for improvement of new and targeted therapeutic approaches. The use of altered PFAAs profile may explain pathogenesis and severity of epilepsy. They also, may act as prognostic factor for the frequency of fits and status epileptics in PRE patients, raising the question of whether such findings had an effect in frequency of PRE fits.

Conflict of Interest: The authors declare that they have no conflict of interest.

Acknowledgements:Authors of this paper sincerely acknowledge Grant office at Assiut medical school for funding this work.

\section{REFERENCES}

1. Thijs RD et al. (2019):Epilepsy in adults. The Lancet, 393(10172): 689-701.

2. Kobow K I, Blümcke I (2018):Epigenetics in epilepsy. Neuroscience Letters, 667: 40-46.

3. St. Louis EK, Cascino G D (2016):Diagnosis of Epilepsy and Related Episodic Disorders. CONTINUUM: Lifelong Learning in Neurology, 22(1) 15-37.

4. Alexopoulos AV (2013): Pharmacoresistant epilepsy: Definition and explanation. Epileptology, 1(1): 38-42.

5. Kwan $P$ (2010):Definition of drug resistant epilepsy: consensus proposal by the adhoc Task Force of the ILAE commission on therapeutic strategies. Epilepsia, 51(6) 10691077.

6. Shorvon S, Tomson T (2011):Sudden unexpected death in epilepsy. The Lancet, 378(9808):2028-2038.

7. Westmark CJ (2018): A role for amino acid balance in dietary treatments for epilepsy. The Journal of nutrition,148(3) :307308.

8. Czapinski P, Blaszczyk B, Czuczwar S J (2005): Mechanisms of action of antiepileptic drugs. Current Topics in Medicinal Chemistry, 5(1) :3-14.

9. Meldrum BS (2000): Glutamate as a neurotransmitter in the brain: review of physiology and pathology. The Journal of Nutrition, 130(4) :1007S-1015S.

10. López-Corcuera B, Geerlings A, Aragón C (2001):Glycine neurotransmitter transporters:anupdate. Molecular Membrane Biology, 18(1): 13-20.
11. Tyagarajan SK, Fritschy JM(2014): Gephyrin: a master regulator of neuronal function? Nat Rev Neurosci, 15(3) :14156.

12. Gruenbaum SE., Roni D, Amedeo R, Hitten PZ, Amber T, Nihal dL, Tore E. (2019):Effects of Branched-Chain Amino Acid Supplementation on Spontaneous Seizures and Neuronal Viability in a Model of Mesial Temporal Lobe Epilepsy, journal of Neurosurgical Anesthesiology, 31 - (2) 247-256.

13. Hrncic D (2018): Sulfur Containing Amino Acids in Seizures: Current State of the Art. Current Medicinal Chemistry, 25(3): 378-390.

14. Rainesalo S (2004): Plasma and cerebrospinal fluid amino acids in epileptic patients. Neurochemical research, 29(1): 319-324.

15. Louzada $P$ (2004): Taurine prevents the neurotoxicity of $\beta$ amyloid and glutamate receptor agonists: activation of GABA receptors and possible implications for Alzheimer's disease and other neurological disorders. The FASEB Journal, 18(3):511518.

16. Lee WT (2011): Disorders of amino acid metabolism associated with epilepsy. Brain and Development, 33(9): 745-752.

17. Giussani G (2016): A population-based study of active and drug-resistant epilepsies in Northern Italy. Epilepsy \& Behavior, 55:30-37.

18. Saleem T, Dahpy MA, Ezzat G, Abdelrahman G, AbdelAziz E, Farghaly R(2019): The Profile of Plasma Free Amino Acids in Type 2 Diabetes Mellitus with Insulin Resistance: Association with Microalbuminuria and Macroalbuminuria. Applied Biochemistry and Biotechnology, 188(3):854-867.

19. Huxtable R et al. (1983):Epilepsy and the concentrations of plasma amino acids in humans. Neurochemistry international, 5(1) :125-135.

20. Janjua NA, Kabuto H, Mori A (1992):Increased plasma glutamic acid in a genetic model of epilepsy. Neurochemical research, 17(3): 293-296.

21. Löscher W(1993):Determination of GABA and vigabatrin in human plasma by a rapid and simple HPLC method: correlation between clinical response to vigabatrin and increase in plasma GABA. Epilepsy Research, 14(3): 245-255.

22. Evangeliou A (2009): Branched chain amino acids as adjunctive therapy to ketogenic dietin epilepsy: pilot study and hypothesis. Journal of Child Neurology, 24(10): 1268-1272.

23. Suárez LM (2016): Taurine content in different brain structures during ageing: effect on hippocampal synaptic plasticity. Amino Acids, 48(5): 1199-1208.

24. Gietzen DW (2018):Indispensable amino acid-deficient diets induce seizures in ketogenic diet-fed rodents, demonstrating a role for amino acid balance in dietarytreatments for epilepsy. The Journal of Nutrition, 148(3): 480-489.

25. Carlson H (1992): Seizure related elevations of extracellular amino acids in human focal epilepsy. Neuroscience letters, 140(1): 30-32.

26. De Sarro G(1993):L-Arginine potentiates excitatory amino acid-induced seizures elicited in the deep prepiriform cortex. European journal of pharmacology, 230(2): 151-158.

27. Kobow K, Blumcke I (2017): Epigenetics in epilepsy. Neuroscience Letters, 22; 667: 40-46. 